\title{
Worms gang up on bacteria
}

The nematode worm Caenorhabditis elegans can be shy or gregarious when feeding time arrives. New work uncovers some of the neurons and genes that are involved in regulating social feeding behaviour in the worm, and points to multiple systems of antagonistic signalling that control whether, and when, the worms aggregate into feeding groups.

The standard laboratory strain of C. elegans is a loner, preferring solitary feeding. But worms with a valine-tophenylalanine mutation at residue 215 of NPR-1 - a putative G-proteincoupled receptor - come together to form aggregates when they encounter bacteria (their food source). And $n p r-1$-knockout worms also aggregate, indicating that the form of the receptor that contains valine (NPR-1 215V) normally suppresses aggregation. In two studies, de Bono and colleagues take advantage of the small nervous system of the worm and the powerful genetic tools available to delve deeper into the control of social feeding.

The first study investigated how and where NPR-1 acts. They constructed a transgene that expressed NPR-1 215V that was tagged with green fluorescent protein (GFP) and driven by the npr-1 promoter. When this transgene was expressed in $n p r-1$ knockout worms, it suppressed aggregation, and the worms showed GFP expression in a number of neurons. By using different promoters to drive expression of the transgene in subsets of neurons, the authors showed that expression in just three sensory neurons - AQR, PQR and URX - was sufficient to suppress aggregation.

These three neurons are exposed to the fluid in the body cavity. Their firing can be inhibited by the selective expression of a gain-of-function mutant of a potassium channel, EGL-2, and this also suppresses aggregation. The ability of these neurons to mediate social feeding seems to depend on signalling through a cyclic-GMP-gated ion channel, as neuron-specific mutations in $\operatorname{tax}-2$ or tax-4, which encode the subunits of

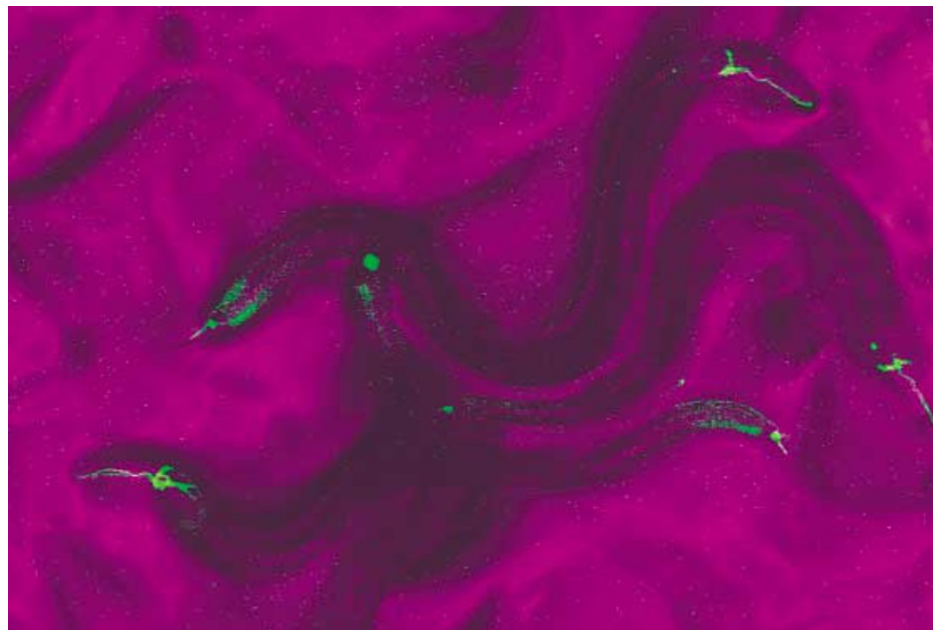

GFP expression in the body-cavity neurons of $C$. elegans. Courtesy of S. Reichelt, MRC-LMB, Cambridge, UK.

this channel, also inhibited aggregation. So, it seems that NPR-1 suppresses aggregation by antagonizing signalling through TAX-2 and TAX-4 in these sensory neurons.

The second study investigated how external stimuli might elicit aggregation. A screen for mutations that interfered with aggregation in $n p r$-1-null animals identified four genes. Two of these, osm-9 and ocr-2, are thought to encode subunits of a TRP (transient receptor potential)-related cation channel in C. elegans chemosensory neurons, and are required for avoidance of various noxious stimuli. The other two genes, $o d r-4$ and $o d r-8$, are required to localize some chemosensory receptors to sensory cilia.

Localized expression of minigenes in specific neurons of doubleknockout animals showed that the expression of $o c r-2$ and $o d r-4$ is required in specific nociceptive neurons to rescue social feeding. Laser ablation of these neurons (the ASH and ADL pairs) abolished social feeding behaviour. Another piece of the puzzle came from studies of worms in which the osm-3 kinesin was knocked out. This gene product is required for the correct formation of sensory cilia on ASH and ADL, and on other sensory neurons. Although knocking out osm-3 interferes with the development of ASH and ADL, it doesn't suppress social feeding. The authors propose that, as well as blocking the ability of $\mathrm{ASH}$ and ADL to promote social feeding, lack of OSM-3 blocks antagonistic signals that normally inhibit this behaviour. In support of this idea, removing OSM-3 restored social feeding to animals defective in ODR-4 or OCR-2. So, as with the body-cavity neurons, nociceptive sensory neurons might be involved in a system of antagonism between signals that promote aggregation and those that suppress it.

As these neurons are required for responses to stressful or aversive stimuli, de Bono et al. propose that aggregation is a response to an aversive stimulus that is produced by bacteria. The next step will be to find out what the aversive stimulus that promotes aggregation is - and how these different control systems interact to determine when social feeding occurs.

(2) References and links Rachel Jones ORIGINAL RESEARCH PAPERS de Bono, $\mathrm{M}$ et al. Social feeding in Caenorhabditis elegans is induced by neurons that detect aversive stimuli. Nature 419, 899-903 (2002) | Coates, J. C. \& de Bono, M. Antagonistic pathways in neurons exposed to body fluid regulate social feeding in Caenorhabditis elegans. Nature 419, 925-929 (2002)

FURTHER READING Rankin, C. H. From gene to FURTHER READING Rankin, C. H. From gene
identified neuron to behaviour in Caenorhabditis elegans. Nature Rev. Genet. 3, 622-630 (2002)

\section{IN THE NEWS}

Lorenzo's oil

After years of controversy, the unorthodox treatment that inspired the1992 film

Lorenzo's Oil has been shown to work. Lorenzo Odone was five years old when he first developed symptoms of the rare genetic condition adrenoleukodystrophy (ALD). He was given two years to live. Determined to find a cure, his parents delved into the medical and scientific

literature, and came up with 'Lorenzo's oil', a mixture of oleic and erucic acids. At 24, Lorenzo is still alive today, but the medical community has always been sceptical about whether the oil actually works. Hugo Moser of the Kennedy Krieger Institute in Baltimore recently unveiled the findings of a ten-year trial of the effects of Lorenzo's oil in boys with early-stage ALD. The oil reverses the main biochemical abnormality of ALD - the accumulation of very-long-chain fatty acids. This study confirms that it can also markedly reduce the chances of developing the devastating neurological symptoms, which reflect a progressive loss of myelin. According to lan Duncan of the University of Wisconsin, the consensus among specialists is that "this is an extremely important finding .. [most specialists] now acknowledge that this therapy can work" (The Guardian, UK, 15 October). But the timing of treatment is crucial. "Give the oil as soon as you know your son carries the genetic defect. If you wait, the symptoms might come and then you are in a different ballpark",

Lorenzo's father, Augusto Odone, told the New Scientist (UK, 26 September). "It's not the final cure of the disease", says Ronald Wanders of the University of Amsterdam, "But it at least gives additional time to come up with alternative therapies" (USA Today, 22 October). In the meantime, Moser says, "it's important that we let families and the scientific community know that this positive response has been observed".

Rebecca Craven 